\title{
Ultrasonic Measurement of Lubricant Film Thickness Distribution of Journal Bearing
}

\author{
Wu Ouyang, ${ }^{1,2 a), b)}$ Zhenjiang Zhou, ${ }^{1}$ Yong Jin, ${ }^{1}$ Xinping Yan ${ }^{1,2}$, and Yuanchang Liu ${ }^{3}$ \\ ${ }^{I}$ School of Energy and Power Engineering, Wuhan University of Technology, Wuhan 430063, China \\ ${ }^{2}$ Reliability Engineering Institute, National Engineering Research Center for Water Transport Safety, Wuhan 430063, China \\ ${ }^{3}$ Department of Mechanical Engineering, University College London, London WC1E 7JE, UK
}

(Received 12 March 2020; accepted XXXXX; published online XXXXX)

\begin{abstract}
Most film thickness measurement methods have the defects of damaging the working surface of bearing and cannot measure the minimum film thickness, which makes it difficult to reveal lubrication state and warn of wear. Two non-intrusive ultrasonic methods for measuring film thickness distribution of bearing were proposed, i.e. the full circumferential measurement and the prediction based on limited measuring points. The ultrasonic recognition model of film thickness was built. A film thickness measuring device and its calibration device were constructed. A calibration experiment in the range of $1 \sim 150 \mu \mathrm{m}$ and a measurement experiment of bearing's film thickness distribution were carried out. The results showed that in the calibration range, the relative error of most recognition values was less than $\pm 5 \%$, and some are less than $3 \%$. The identification accuracy of the spring model has zoned phenomenon. The relative difference between the experimental and the simulated values of film thickness was less than $8 \%$ under most working conditions. The predicted values of eccentricity, attitude angle and minimum film thickness have small difference from the simulated values, indicating that the accuracy of the measurement method is high.
\end{abstract}

\section{INTRODUCTION}

Sliding bearing has the characteristics of carrying large impact load, strong ability to absorb vibration, and high operation accuracy. It has become one of the key components of important equipment such as large thermal power and hydroelectric generating units, ship propulsion systems, high-speed machine tools, and main circle pumps of nuclear power plant. Sliding bearing uses fluid film force to float rotating part to avoid direct contact between the friction pair. Therefore, the quality of lubricant film determines the bearing's load carrying capacity, operation stability and service life, which are treated as the critical design and evaluation parameters. ${ }^{1}$ The bearing with harsh working conditions and special materials, such as the water-lubricated rubber bearings for ship propulsion systems with low-viscosity, works at low-speed, cantilever eccentric load from propeller, and intrusion of mud and sand, which is often in thin film lubrication and mixed lubrication. Due to the lack of accurate information of lubrication status, the current lubrication theory cannot well simulate the actual situation, ${ }^{2}$ and poor lubrication often leads to wear, ${ }^{3}$ abnormal friction noise, ${ }^{4}$ shaft vibration $^{5}$ and other accidents. Therefore, it is of great significance for the development of lubrication theory and maintenance to carry out the research on measurement technology of lubricant film thickness of sliding bearing.

Researchers have proposed many film thickness measurement methods based on electrical, magnetic, acoustic, and optical characteristics. These methods have their own applicable scope and limitations. Resistance

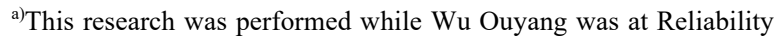
Engineering Institute, School of Energy and Power Engineering, Wuhan University of Technology, Wuhan 430063, China.

${ }^{b)}$ Author to whom correspondence should be addressed. Electronic mail: ouyangw@whut.edu.cn.
}

method $^{6}$ and capacitance method based on the electrical characteristics require establishing a conductive circuit between bearing's friction pairs. This method cannot be used for non-metallic bearing. Raman spectroscopy ${ }^{7}$ and optical interference method ${ }^{8}$ have high measurement accuracy, but only the film thickness in a small area can be measured. Laser-induced fluorescence (LIF) is a lightexcited visualization measurement technology based on fluorescent dyes or group. ${ }^{9}$ Liang et al. first applied LIF to measure the film thickness distribution of water-lubricated thrust bearings. ${ }^{10}$ These methods based on the light principle require that at least one side of friction pairs is a light-transmitting material, but most of bearings are not satisfied. The eddy current sensor method is simple to implement, ${ }^{11}$ but the measurement hole will damage the working surface of bearing, and measurement accuracy is limited by sensor accuracy.

Strong penetrating ability of ultrasonic can prevent damaging the interface of friction pairs, so a non-intrusive ultrasonic method has been developed. ${ }^{12}$ At present, a variety of ultrasonic film thickness recognition models have been developed with each model having a different recognition range of film thickness. Pialucha et al. defined the resonance frequency and a resonant model was formed, which was generally considered to be suitable for measuring the film thickness above $10 \mu \mathrm{m} .{ }^{13}$ Zhang et al. demonstrated that the resonant model could also measure the film thickness range above $100 \mu \mathrm{m} .{ }^{14}$ Dwyer-Joyce et al. adopted the amplitude and phase of a reflected echo to characterize the film thickness for the first time, avoiding measuring the reference signal before each measurement. ${ }^{15}$ 
In 2004, Dwyer-Joyce et al. investigated the potential of the spring model in micro distance measurement, making ultrasonic method able to measure the distance below 1 $\mu \mathrm{m} .{ }^{16}$ Later, they carried out an experimental research of mixed lubrication state on a test rig of steel ball contacting plane. ${ }^{17}$

However, the above ultrasonic measurement experiments are scattered and lack of verification of application range of each model on the same calibration device. Some scholars are exploring a wider range of recognition models, ${ }^{18}$ but these film thickness measurement methods only arrange one or several measuring points making it difficult to obtain more important information such as the minimum film thickness and the film thickness distribution, so the lubrication state of the whole bearing cannot be assessed. Therefore, in this paper, two methods for measuring the film thickness distribution of journal bearing are proposed, the film thickness identification model is built, a film thickness measurement device and a calibration rig are constructed, and a calibration experiment and a measurement experiment of bearing's film thickness distribution are carried out. This method can solve the problem of obtaining the key information of film thickness.

\section{METHODS FOR MEASURING THE FILM THICKNESS DISTRIBUTION OF JOURNAL BEARING}

When bearing works, a film gap is formed between the bearing and the shaft, as shown in Figure 1. Along the direction of rotation, the film thickness changes gradually. For hydrodynamic sliding bearings, they have a wedgeshaped lubricant film thickness distribution, and the minimum film thickness ranges from about $5 \mu \mathrm{m}$ to 100 $\mu \mathrm{m}$. Ultrasonic measurement methods of film thickness use ultrasonic reflection signals on the upper and lower interfaces of the lubricant film to obtain the film thickness.

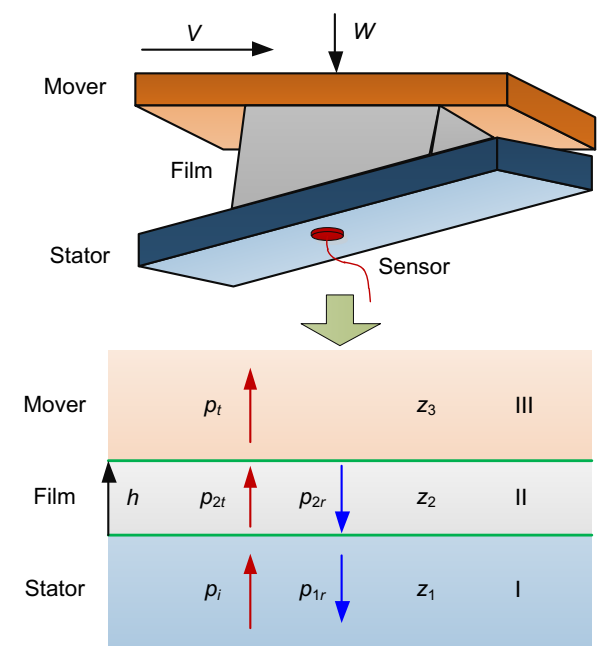

FIG. 1. Vertical incidence of ultrasonic to a three-layer structure

Assume that the thickness of the bearing film layer is $h$, the acoustic impedances of the materials on both sides of the lubrication layer are $z_{1}$ and $z_{3}$, and the acoustic impedance of the film layer is $z_{2}$. When a series of plane waves $\left(p_{i}, v_{i}\right)$ enter the upper interface of film layer perpendicularly, as shown in Figure 1, part of the wave is reflected back to medium I, forming a reflected wave $\left(p_{1 r}, v_{1 \mathrm{r}}\right)$. The other part is transmitted into film layer II, which is $\left(p_{2 t}, v_{2 t}\right)$. When the wave continues to reach the lower interface of film layer, a part of wave $\left(p_{2 r}, v_{2 r}\right)$ is reflected back to the film layer, and the rest wave penetrates into medium III which is denoted as $\left(p_{t}, v_{t}\right)$.

\section{Measurement scheme}

In order to obtain the film thickness distribution of journal bearing, two schemes are proposed.

(1) Full circumferential measurement

As shown in Figure 2, ultrasonic sensors are installed into a hollow shaft, and the signal wire is connected to a pulse generator after passing through the shaft hole. The pulse generator sends a voltage pulse to the ultrasonic sensor, and then receives a reflected signal from the sensor. A wireless measurement module transmits the reflected signal to a data acquisition module, and the signal finally reaches a signal analysis module. When sensors rotate with the rotating shaft, a full circumferential film thickness distribution can be obtained. In the measurement system, a pulse generator is made into a short axis, and needs to undergo a dynamic balance test. Wireless transmission of signal and wireless power supply of pulse generator adopt the induction principle to ensure the transmission stability. The advantage of this method is that it can obtain full circumferential film thickness distributions in real time without considering the influence of factors such as bearing deformation, and without bringing any damage to the integrity of friction pairs. However, this method requires high precision and stability of wireless telemetry.

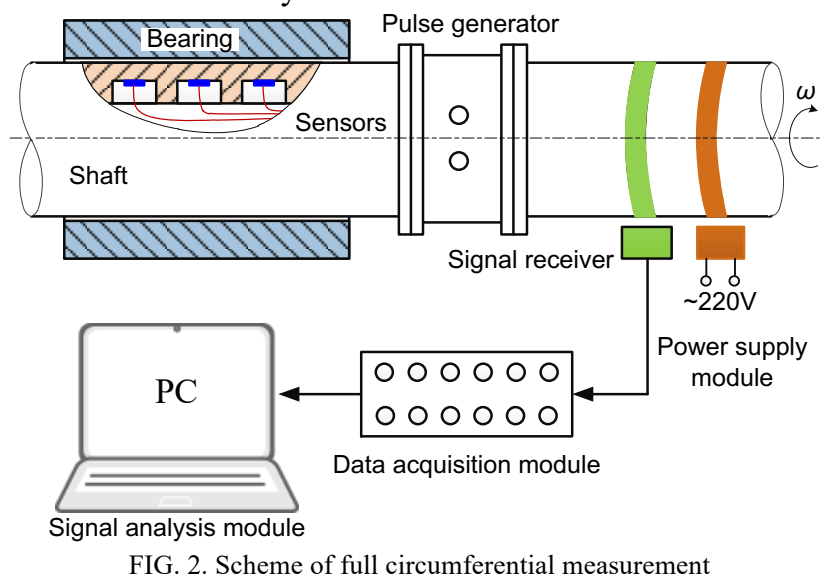

(2) Prediction based on limited measuring points

The film thickness equation of bearing in service can be established:

$$
h=c_{b}\left[1+\varepsilon \cos \left(\varphi-\theta_{0}\right)\right]+\delta+\kappa
$$

Where, $c_{b}$ is the radius clearance of bearing. $\varepsilon$ is the eccentricity. $\theta_{0}$ is the attitude angle. $\varphi$ is the circumferential angle. $\delta$ and $\kappa$ are the deformation and 
wear of bearing lining respectively. $c_{b}$ can be measured after installing of bearing. $\theta_{0}, \varphi$ and $\delta$ will change with the working condition. Due to the slow change of wear, $\kappa$ can be regarded as a fixed value in a period of time. An ultrasonic sensor is installed at position $\varphi$, and measure the film thickness at this position. Four equations are needed to solve $\varepsilon, \theta_{0}, \delta$ and $\kappa$.

As shown in Figure 3, along the circumferential direction, some sensors are arranged on bearing's outer wall, and equation (1) can be established. After these unknown parameters are solved, the film thickness distribution can be obtained and the minimum film thickness $h_{\min }$ can be identified. When the film is thick and the bearing material is hard, the bearing deformation and wear are neglected, and then only two equations are needed to identify the film thickness distribution.

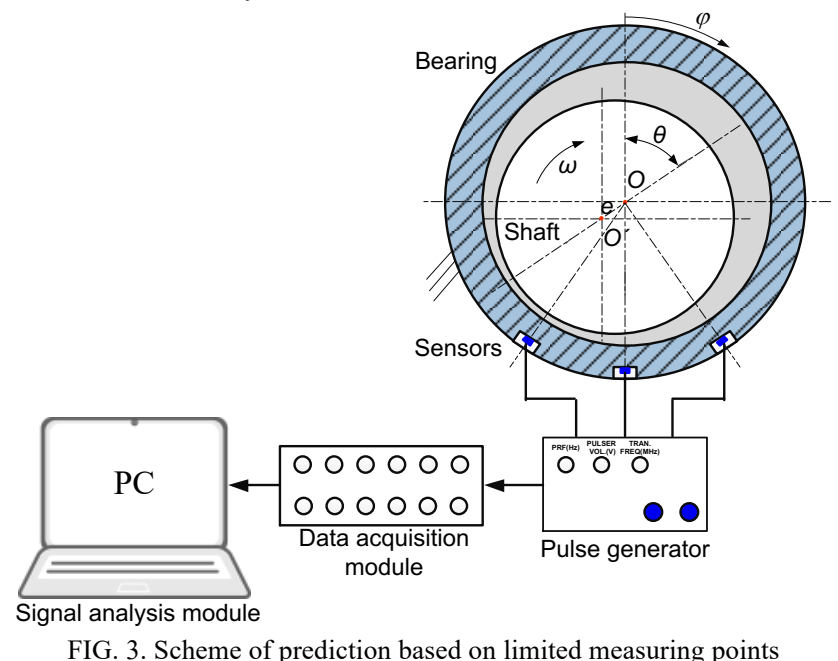

\section{Recognition model of film thickness}

Different thicknesses have different characteristics of ultrasonic reflection signals, based upon which different recognition models of film thickness can be developed. The time flight model uses the time of ultrasonic travelling back and forth in film to convert thickness, which is suitable for large thickness and not suitable for most bearing film thickness. This paper focuses on the situation that ultrasonic round-trip signals are difficult to separate in time domain.

(1) Resonant model

For the ultrasonic generated by a narrow pulse excitation, the frequency spectrum contains multiple frequency components. When film thickness is an integral multiple of the half wavelength of a certain frequency component in the ultrasonic broadband, the minimum value of frequency spectrum will appear at this frequency point. The relationship between film thickness and the frequency corresponding to the minimum value is shown as: ${ }^{14}$

$$
h=\frac{n c}{2 f_{n}}
$$

Where, $n$ is the order of resonance frequency. $f_{n}$ is the $n$-th order resonance frequency. $c$ is the propagation speed of ultrasound in the film.

In order to facilitate measurement, the 1 st order resonance frequency is often selected. When the ultrasound frequency $f$ is $\omega / 2 \pi$, and $f=f_{n}$, the reflection coefficient is obtained:

$$
R=\frac{z_{1}-z_{3}}{z_{1}+z_{3}}
$$

When the film thickness is within the applicable range of the resonant model, the frequency corresponding to the minimum point of reflection coefficient is the resonance frequency, and then the film thickness is calculated. Set the resolution of signal frequency as $\Delta f$ and $m=1$, when resonance frequency is $f$, the film thickness is:

$$
h=h(f)=\frac{c}{2 f}
$$

When resonance frequency is $f+\Delta f$, the film thickness is:

$$
h^{\prime}=h(f+\Delta f)=\frac{c}{2(f+\Delta f)}
$$

The difference between $h^{\prime}$ and $h$ is the minimum resolution of thickness:

$$
\Delta h=h(f+\Delta f)-h(f)=\frac{c \Delta f}{2 f(f+\Delta f)}
$$

According to formula (6), the resolution is related to the frequency within the effective bandwidth of ultrasound. As the frequency increases, the resolution gradually increases as well as the measurement accuracy. The resonant model recognizes the film thickness through the echo resonance between film interfaces, and the frequency of signal spectrum determines the resolution of film thickness. When the film thickness is very thin, high ultrasonic frequency is required, which will cause serious signal attenuation, therefore, the resonant model can only identify relatively thick film thickness.

According to experiment results, when the film thickness is large, the reflection coefficient of ultrasonic on lubricating layer changes periodically with frequency and the reflection coefficient will have multiple minimum values. At this time, the relationship between the film thickness and the interval $\Delta f_{n}$ between two minimum values can be calculated as: ${ }^{15}$

$$
h=\frac{c}{2 \Delta f_{n}}
$$

Formula (4) is very similar to formula (7). When film thickness is thin, there is usually only a 1-order minimum value in the frequency bandwidth of ultrasonic reflection signal, and the film thickness is recognized by formula (4). When film thickness is thick, there will be multiple minimum points in the frequency bandwidth, and film thickness is recognized by formula (7).

(2) Spring model 
When the film thickness is much smaller than the half wavelength of ultrasonic $(\mathrm{h}<<\lambda)$, reflected signals of the upper and lower interfaces of lubricant film almost completely overlap, and it is difficult to directly recognize the film thickness from the echo signal. Stiffness $K$ of liquid film determines the reflection coefficient $R$ of incident wave passing through liquid film as: ${ }^{19}$

$$
R=\frac{z_{1}-z_{3}+\mathrm{i} \omega\left(z_{1} z_{3} / K\right)}{z_{1}+z_{3}+\mathrm{i} \omega\left(z_{1} z_{3} / K\right)}
$$

Where, $\omega$ is ultrasonic angular frequency. The relationship between the thickness of lubricant film layer, the reflection coefficient, and the material parameters of each layer is:

$$
h=\frac{\rho c^{2}}{\omega z_{1} z_{2}} \sqrt{\frac{R^{2}\left(z_{1}+z_{2}\right)^{2}-\left(z_{1}-z_{2}\right)^{2}}{1-R^{2}}}
$$

Where, $\rho$ is the density of liquid film. First, the reflection e cho of stator layer (bearing bushing) at air is measured as $r$ eference signal, and then approximate reflection coefficien $\mathrm{t}$ is obtained by comparing amplitude of reflected signal $\mathrm{fr}$ om liquid film with that of reference signal, and eventually film thickness is calculated.

\section{THE DESIGN OF FILM THICKNESS MEASURING DEVICE AND CALIBRATION EXPERIMENT}

\section{Lubricant film thickness measuring device}

The lubricant film thickness measuring device of bearing consists of two parts, i.e. hardware and software components. The hardware part is composed as shown in Figure 3, which mainly includes sensors, a pulse generator, an oscilloscope, and a signal analysis module. Ultrasonic sensor is a PZT5A1 circular ultrasonic piezoelectric element (Morgan Advanced Materials, UK), with a diameter of $7 \mathrm{~mm}$ and a thickness of $0.2 \mathrm{~mm}$. The pulse generator is selected based on the frequency of ultrasonic sensors and in this design 5072pr (Olympus Corporation, Japan) is used. Its pulse shape is a negative square wave pulse with a maximum pulse repetition frequency of 20 $\mathrm{kHz}$. The amplitude of the excitation pulse can be adjusted based upon specific needs, and the excitation pulse width is adjusted according to the ultrasonic center frequency and the adhesive quality of sensor. The oscilloscope is used to collect reflected signals received by the pulse generator. Basic parameters of the DS2102A digital oscilloscope (RIGOL Technology Co., Ltd., China) are: $100 \mathrm{MHz}$ broadband, 2-channel, and sampling rate is $2 \mathrm{GS} / \mathrm{s}$

The software part is to solve the above recognition models to obtain film thickness. First, the reflected signal is processed to obtain the frequency domain of reflection coefficient. Then the appropriate recognition model is selected according to the characteristics of reflection coefficient curve as:

(1) If reflection coefficient is a smooth increasing curve with the increase of frequency, the spring model is used;
(2) If reflection coefficient increases with frequency and there is a minimum point or multiple minimum points, the resonant model is selected.

\section{Calibration device}

In order to verify the recognition accuracy of film thickness measuring device, a calibration device needs to be designed, which can provide a known standard film thickness, use the measuring device to recognize film thickness, and compare recognition value with standard value to determine the recognition accuracy. The two most important features of this calibration device are: the measurement plane has high levelness, and the gap at the micron level can be adjusted to simulate film thickness.

A vertical calibration device is designed, as shown in Figure 4. The device includes: micrmeter (Type 153-301, Mitutoyo Corporation, Japan), test module, upper plate, lower plate, baseplate, column and adjustable shore. The micrmeter has high accuracy, its adjustment range is 0 $25 \mathrm{~mm}$, and the accuracy is $0.5 \mu \mathrm{m}$. It is required to ensure that the axis of the micrmeter is perpendicular to the lower plate, and four shores are used to adjust the level of the upper surface of the lower plate. Test module includes a rotating block and a static block. Among them, the rotating block is installed on a micrmeter, which rotates with the shaft to simulate the mover of bearing. The static block is installed on a lower plate, and the upper surface of the static block is bonded with a plexiglass ring, which is used to store liquid. An ultrasonic sensor is attached to the lower surface of the static block. The static block simulates the stator of bearing.

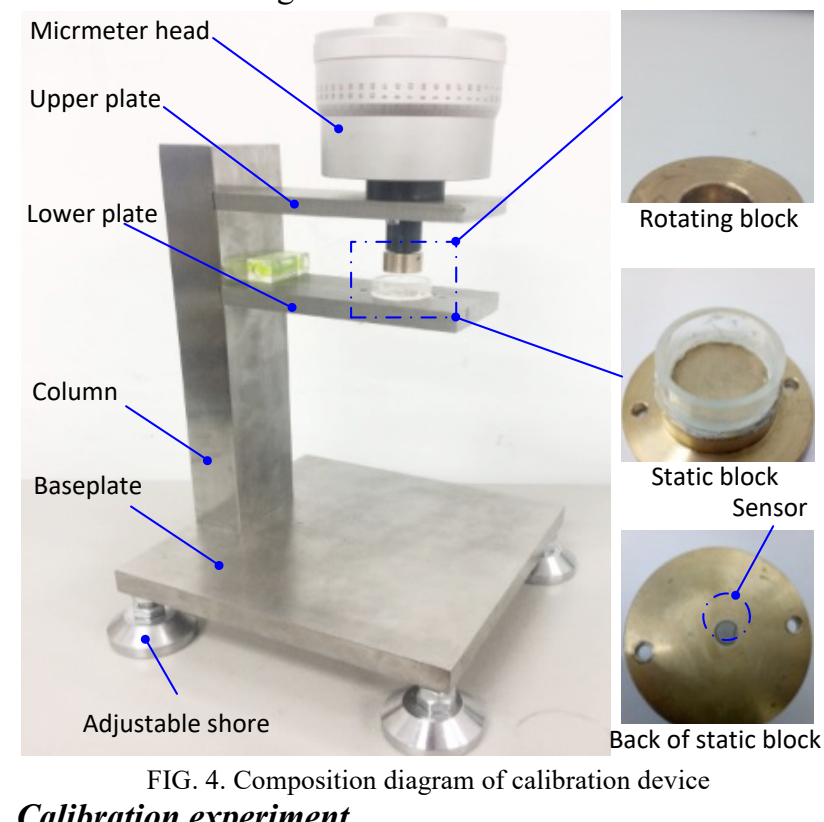

\section{Calibration experiment}

In the calibration experiment, brass and 304 stainless steel are used for the rotating block and the static block, respectively, that is, the test module is a copper-oil-copper system and steel-oil- steel system. Brand of lubricating oil is ISO VG46. The acoustic impedance of brass, stainless steel and oil is $3.96 \times 10^{6} \mathrm{~g} /\left(\mathrm{cm}^{2} \mathrm{~s}\right) 4.53 \times 10^{6} \mathrm{~g} /\left(\mathrm{cm}^{2} \mathrm{~s}\right)$, and $0.1 \times 10^{6} \mathrm{~g} /\left(\mathrm{cm}^{2} \mathrm{~s}\right)$ respectively. The propagation speed of 
ultrasound in stainless steel, brass and oil is $6.2 \mathrm{~km} / \mathrm{s}, 4.7$ $\mathrm{km} / \mathrm{s}$ and $1.45 \mathrm{~km} / \mathrm{s}$ respectively.

(1) $10 \mu \mathrm{m} \sim 150 \mu \mathrm{m}$

Standard film thicknesses $\left(h_{s}\right)$ in the range of 10 $\mu \mathrm{m} \sim 150 \mu \mathrm{m}$ are constructed by the calibration device, and echo signals of different materials are analyzed using the resonant model, as shown in Figure 5. It can be seen that when film thickness is in the range of $10 \mu \mathrm{m} \sim 100 \mu \mathrm{m}$, relative error in most areas is less than $\pm 5 \%$. When film thickness is less than $25 \mu \mathrm{m}$, relative error is greater than \pm $5 \%$ but less than $\pm 10 \%$. However, when film thickness is $10 \mu \mathrm{m}$, relative error of the copper block reaches $17.7 \%$. When film thickness ranges from $100 \mu \mathrm{m}$ to $150 \mu \mathrm{m}$, relative errors of the recognition values are less than \pm 3 $\%$. It shows that when film thickness is above $10 \mu \mathrm{m}$, the resonant model is suitable for recognizing both small and large thicknesses.

When film thickness ranges from $10 \mu \mathrm{m}$ to $100 \mu \mathrm{m}$, the mean absolute error (MAE) between recognition values of two materials and standard values are $6.50 \mu \mathrm{m}$ and 7.36 $\mu \mathrm{m}$ respectively, indicating that measurement accuracy of copper block is slightly higher than that of stainless steel block. When film thickness ranges from $100 \mu \mathrm{m}$ to 150 $\mu \mathrm{m}$, MAE of two materials are $1.67 \mu \mathrm{m}$ and $0.93 \mu \mathrm{m}$ respectively, indicating that measurement accuracy of stainless steel block is slightly higher than that of copper block. In general, the experiment results of copper block and stainless steel block are slightly different.
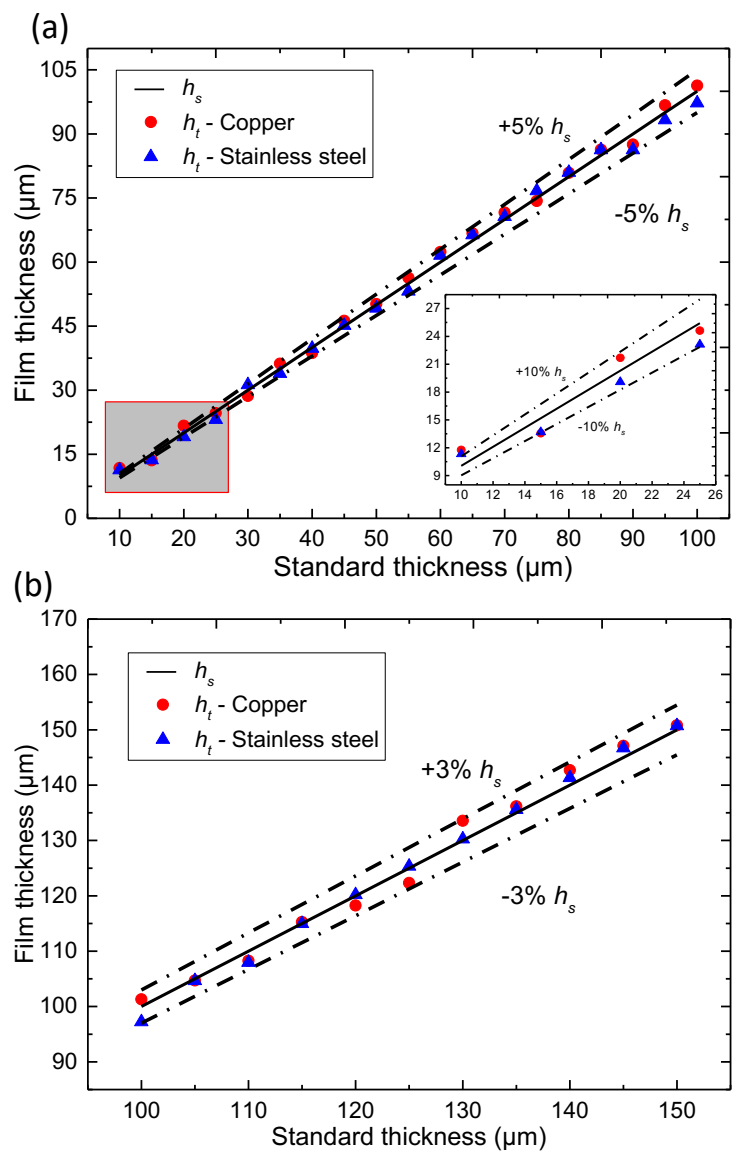

FIG. 5. Film thickness calibration results of two kinds of materials based on the resonance model: (a) $10 \mu \mathrm{m} \sim 100 \mu \mathrm{m}$; (b) $100 \mu \mathrm{m} \sim 150 \mu \mathrm{m}$

(2) $1 \mu \mathrm{m} \sim 10 \mu \mathrm{m}$

Standard film thicknesses of $2.5 \mu \mathrm{m}, 3.5 \mu \mathrm{m}, 4.5 \mu \mathrm{m}$, $6 \mu \mathrm{m}$ and $7.5 \mu \mathrm{m}$ are constructed by the calibration device, and echo signals are analyzed using the spring model, as shown in Figure 6. It can be seen that frequency domain diagram of reflected signal presents a parabola. The larger film thickness, the larger the amplitude of parabola. The amplitude of reflected echo increases with increasing film thickness, and reflection coefficient increases with increasing frequency and film thickness, and tends to 1.

(a)
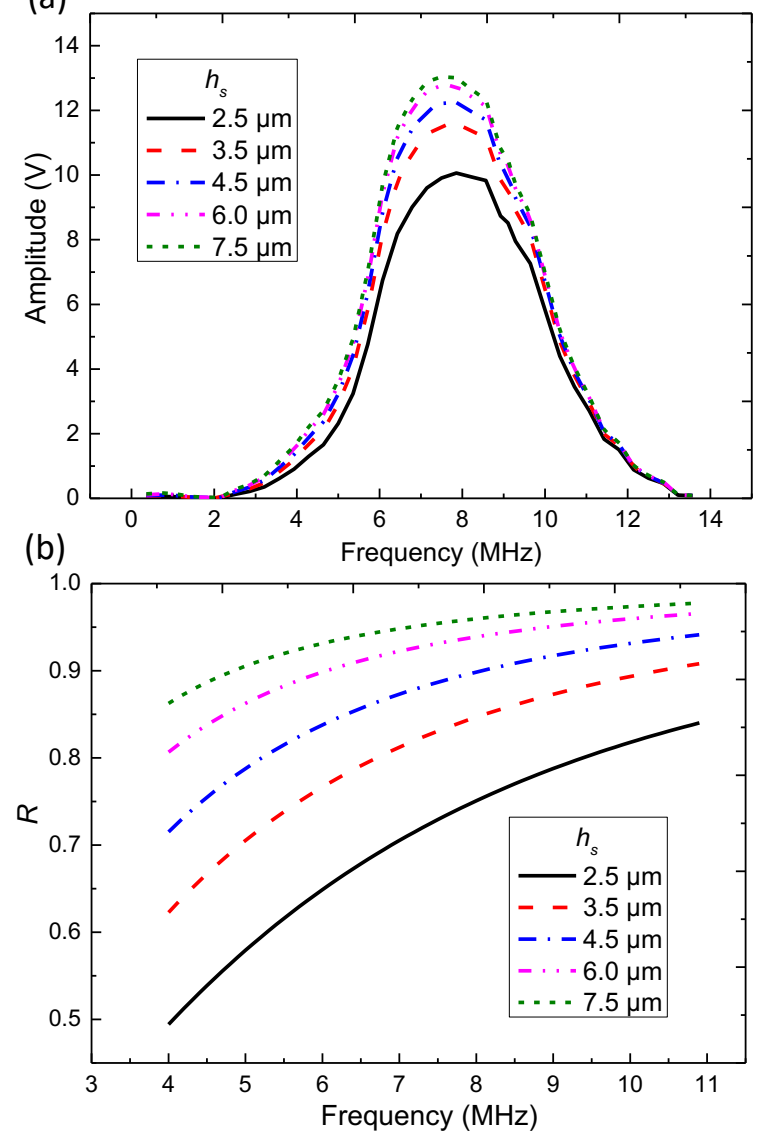

FIG. 6. Ultrasonic echo signals with different film thickness: (a) Frequency domain diagram; (b) Reflection coefficient

Recognition values of film thickness at different frequencies are shown in Figure 7. It can be seen that as frequency changes, recognition value fluctuates near the standard values. The error analysis is shown in Figure 7 (b). With respect to the difference between average of recognition values and standard value, the difference is about $0.18 \mu \mathrm{m}$ when $h_{s}$ is $4.5 \mu \mathrm{m}$ or $6.0 \mu \mathrm{m}$, and the difference in other $h_{s}$ is less than $0.05 \mu \mathrm{m}$. When $h_{s}$ is 7.5 $\mu \mathrm{m}$, the standard deviation (SD) of recognition value is minimum, indicating that test data has the best dispersion, and with the decrease of film thickness, the dispersion increases slightly. SD is maximum when $h_{s}$ is $2.5 \mu \mathrm{m}$. In order to avoid the influence of data measurement unit, coefficient of variation (CV) of tests is analyzed. It can be seen that when $h_{s}$ is $7.5 \mu \mathrm{m}, \mathrm{CV}$ is the minimum. $\mathrm{CV}$ of $h_{s}$ 
at $2.5 \mu \mathrm{m}$ is 9 times higher than that at $7.5 \mu \mathrm{m}$, it also proves that when $h_{s}$ is $2.5 \mu \mathrm{m}$, the test result is affected most by frequency. This is because signal energy at different frequencies is different. The smaller the film thickness is, the smaller the signal to noise ratio of the signal is, which leads to the greater variation of the reflection coefficient with the frequency. According to formula (9), the fluctuation of recognition value of film thickness will also increase. Therefore, in order to reduce the influence of frequency, the recognition values at various frequencies should be averaged.

(a)

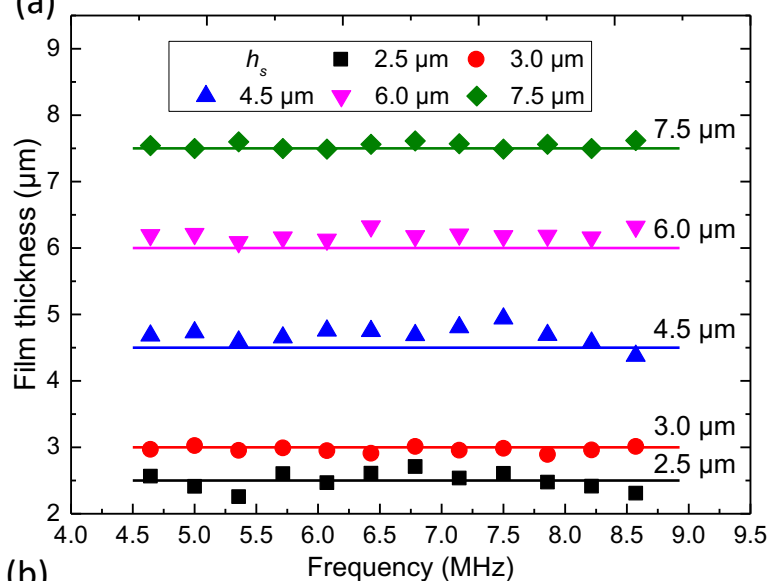

(b)

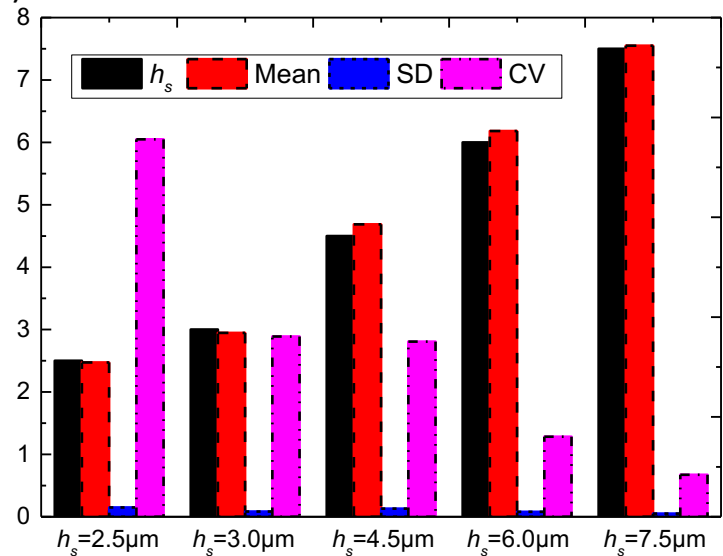

FIG. 7. Identification value of film thickness under different ultrasonic frequencies: (a) Recognition value; (b) Error analysis

$h_{s}$ in the range of $1 \mu \mathrm{m} \sim 10 \mu \mathrm{m}$ are constructed by the calibration device, and echo signals are analyzed using the spring model, as shown in Figure 8. It can be seen that in the range of $2.5 \mu \mathrm{m} \sim 7.5 \mu \mathrm{m}$, recognition value is very close to standard value, and relative error is less than $5 \%$. But outside this area, there are obvious errors between recognition values and calibration values. In 1 \# Area, recognition value is almost kept at $2.3 \mu \mathrm{m}$, which is due to machining errors on the surface of two test blocks, resulting in supporting by rough peaks when the distance between test blocks is less than a certain value, that is, the interface has contact. At this time, although the micrmeter continues to tighten, the distance remains unchanged. It can be imagined that if the roughness of test blocks' surfaces is reduced, recognition value of minimum film thickness will close to $0 \mu \mathrm{m}$.

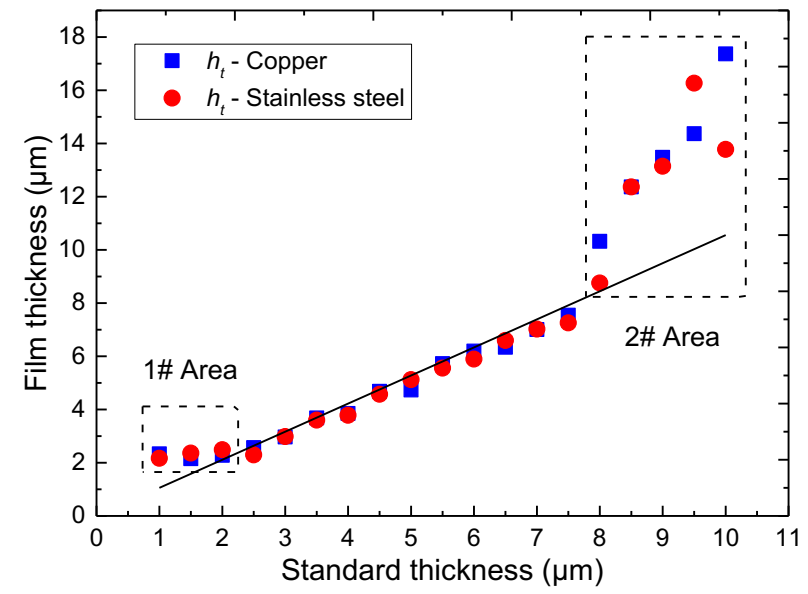

FIG. 8. Film thickness calibration results of two kinds of material test blocks based on spring model

In order to analyze measuring range of the spring model, transmission process of ultrasound in Figure 1 is calculated, and the relationship between sound pressure reflection coefficient, film thickness and frequency can be obtained: 15

$$
R=\sqrt{\frac{\frac{1}{4}\left(n-\frac{1}{n}\right)^{2} \sin ^{2}\left(\frac{2 \pi h}{\lambda}\right)}{1+\frac{1}{4}\left(n-\frac{1}{n}\right)^{2} \sin ^{2}\left(\frac{2 \pi h}{\lambda}\right)}}
$$

Where, $n$ is $z_{1} / z_{2}$. When the material of two test blocks are stainless steel, as shown in Figure 9, the horizontal coordinate is logarithm of the product of film thickness and frequency $(\lg (h \cdot f))$, and the vertical coordinate is the reflection coefficient. If the measurement area of the spring model is $0.1<\mathrm{R}<0.98$, and the product range of film thickness and frequency corresponding to this area is $1 \sim 35 \mu \mathrm{m} \cdot \mathrm{MHz}$. The center frequency of the sensor is 10 $\mathrm{MHz}$, and its $-6 \mathrm{DB}$ broadband is $4.5 \sim 8.5 \mathrm{MHz}$. Therefore, the measurement range of the spring model under the above conditions is $0.11 \sim 7.8 \mu \mathrm{m}$. In addition, when $0.1<\mathrm{R}<0.95$, the measurement range of the spring model is $0.11 \sim 4.8 \mu \mathrm{m}$. Moreover, for the sensor with a center frequency of $10 \mathrm{MHz}$, when film thickness is more than $5 \mu \mathrm{m}$, reflection coefficient of film will be above 0.95. According to formula (10), the larger the film thickness is, the more the denominator tends to 0 , and the more sensitive the film thickness is to the error of reflection coefficient. This reveals the reason for the big recognition error in 2 \# Area theoretically.

In summary, When film thickness is greater than 10 $\mu \mathrm{m}$, the resonant model is suitable for identifying both small and large film thicknesses. When film thickness ranges from $10 \mu \mathrm{m}$ to $100 \mu \mathrm{m}$, the relative error of most of the recognition values is less than $\pm 5 \%$. When it is 100 $\mu \mathrm{m} \sim 150 \mu \mathrm{m}$, the relative error of the recognition value is less than $\pm 3 \%$. The application range of the spring model is affected by center frequency of ultrasonic sensor, which 
results in a zoning phenomenon in its recognition accuracy. The measurement device has a recognition error of less than $5 \%$ in the range of $2.5 \mu \mathrm{m}$ to $7.5 \mu \mathrm{m}$.

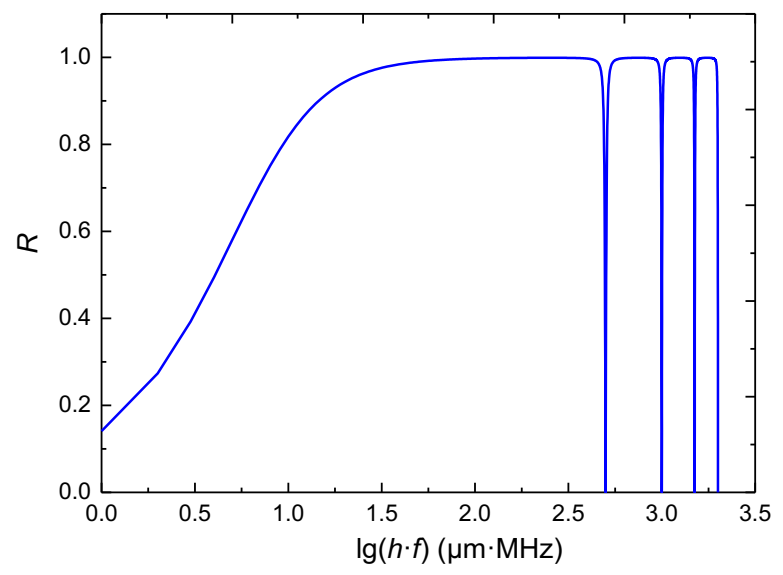

FIG. 9. Relationship between reflection coefficient and $h \cdot f$

\section{BEARING TEST RIG AND TEST BEARING}

\section{Test rig}

The bearing film thickness will be measured on a bearing test rig, ${ }^{20}$ as shown in Figure 10, it includes: a drive module, a test bearing module, a loading module and a measuring module. Power of the drive motor is $600 \mathrm{~W}$, driving torque is transmitted to the test shaft through two $\mathrm{V}$-belts, and the rotational speed of test shaft ranges from 0 to $1500 \mathrm{r} / \mathrm{min}$. The test shaft is supported by two rolling bearings, and the test bearing is suspended above the test shaft. Loading screw applies force downward on the test bearing, and a force sensor is installed between loading screw and test bearing. Feedback control ensures that the bearing receives specified force. An infrared measuring device is installed on the side of the large pulley to measure the rotational speed.

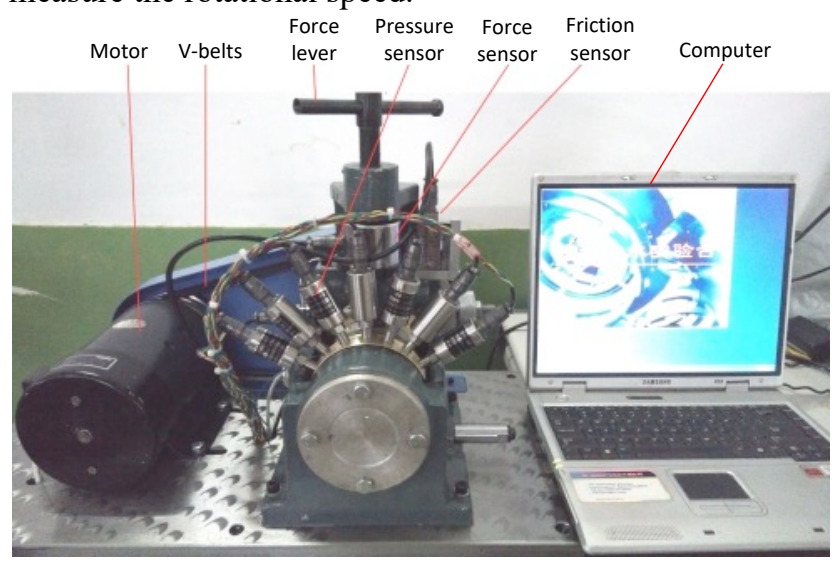

\section{Test bearing}

FIG. 10. Photo of test rig

The test bearing is a half pad with an inner radius of $30 \mathrm{~mm}$, an outer radius of $35 \mathrm{~mm}$, a width of $130 \mathrm{~mm}$, and a bearing radius gap of $0.56 \mathrm{~mm}$. The brand of lubricating oil is ISO VG46, and the inlet oil temperature is $30^{\circ} \mathrm{C}$. As shown in Figure 11, in order to facilitate the installation of sensors, three ultrasonic sensors are arranged at equal intervals on one side of bearing. Before pasting sensors, polish bearing's back to form three platforms firstly. Before pasting, the platform needs to be cleaned with alcohol. The distance from sensor to bearing's inner wall is $3 \mathrm{~mm}$. After pasting, signal wires are welded to the positive and negative of the sensor and then connected to the pulse generator.

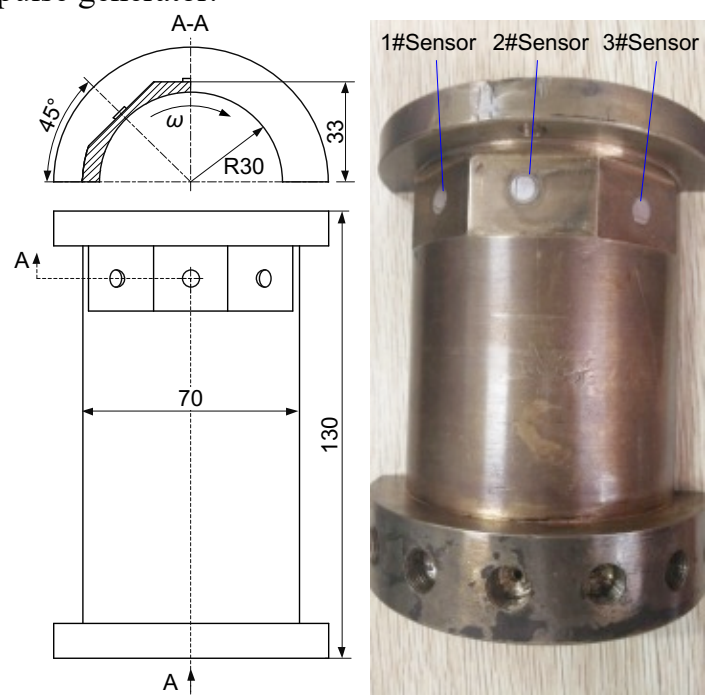

FIG. 11. Arrangement position of sensors on test bearing

\section{RESULTS AND DISCUSSION}

\section{Stability of measurement results}

Due to the manufacturing and installation errors of the test rig, there is a vibration phenomenon of test bearing during experiment. In order to analyze the influence of vibration on test results, multiple measurements under the same working conditions are carried out. Take \# 2 measuring point as an example, load force is $1 \mathrm{kN}$ and rotational speed is $300 \mathrm{r} / \mathrm{min}, 400 \mathrm{r} / \mathrm{min}$ and $600 \mathrm{r} / \mathrm{min}$ respectively. As shown in figure 12 , it can be seen that recognition value fluctuates under different speeds, indicating that bearing vibration will affect the stability of test results.

Take relative differences between the maximum value and minimum value and average value respectively, the relative differences of $300 \mathrm{r} / \mathrm{min}, 400 \mathrm{r} / \mathrm{min}$ and $600 \mathrm{r} / \mathrm{min}$ are $-3.2 \% \sim 3.5 \%,-4.3 \% \sim 4.6 \%$ and $-9.0 \% \sim 5.7 \%$. Compared with SDs at different speeds, it can be seen that $\mathrm{SD}$ is the largest at $600 \mathrm{r} / \mathrm{min}$, followed by SD at 400 $\mathrm{r} / \mathrm{min}$, and SD is the smallest at $300 \mathrm{r} / \mathrm{min}$.

The change law of $\mathrm{CV}$ with speed is exactly opposite to that of SD. CV at $300 \mathrm{r} / \mathrm{min}$ is about 1.66 times of $\mathrm{CV}$ at $600 \mathrm{r} / \mathrm{min}$. It can be seen that the higher rotational speed, the greater discreteness of recognition data. This is because bearing vibration increases as rotational speed increases. However, the higher rotational speed, the larger film thickness, resulting in reduction of relative difference. Therefore, on one hand, adjusting the test rig to minimize its vibration before measuring, on the other hand, the 
average value of multiple measurements can be taken as the final recognition value.

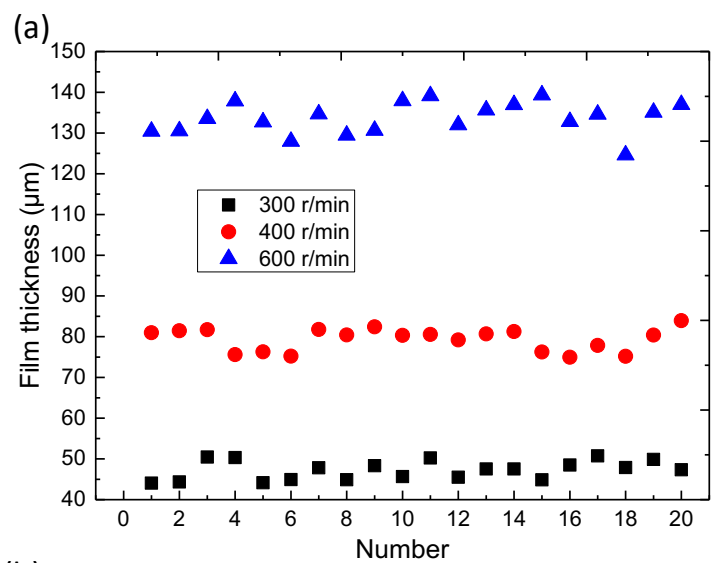

(b)

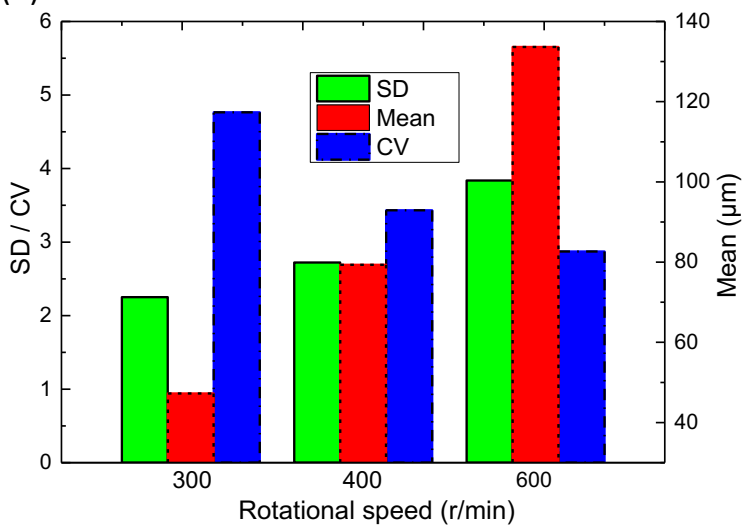

FIG. 12. Multiple measurements at different rotational speeds: (a) 20 times measurement; (b) Discreteness

\section{Comparison of experimental and simulated values of film} thickness

If the test state meets the hydrodynamic lubrication theory, because the theory is relatively mature, accuracy of the above measurement method can be verified by comparing experimental and simulated values of bearing film thickness. In this experiment, the maximum load is 4 $\mathrm{kN}$, the corresponding specific pressure is about $0.51 \mathrm{MPa}$. The load is small, and what's more, the test bearing is metal, so bearing deformation can be ignored and it meets the verification ideas. The simulation method of bearing film thickness: ${ }^{20}$ establish a hydrodynamic lubrication model of journal bearing, and solve the model by using the finite difference method or the finite element method to obtain the film thickness distribution, and extract film thickness values at measurement points.

The load is $2 \mathrm{kN}$, and experimental and simulated values of bearing film thickness at different speeds are shown in Figure 13. It can be seen that the relative difference between experimental and simulated values is less than $8 \%$ under most speeds. The difference of 1 \# measuring point is the smallest, and its relative difference is less than $4 \%$ at most speeds, when rotational speeds are $350 \mathrm{r} / \mathrm{min}, 400 \mathrm{r} / \mathrm{min}$ and $450 \mathrm{r} / \mathrm{min}$, the relative difference is about $1.5 \%$. The difference of $2 \#$ measuring point is the largest, when rotational speeds are $350 \mathrm{r} / \mathrm{min}, 400$ $\mathrm{r} / \mathrm{min}$ and $450 \mathrm{r} / \mathrm{min}$, the relative difference is about $8 \%$, but at other speeds, the relative difference is about $14 \%$. Moreover, the relative difference of these two measuring points at high speed is obviously smaller than at low speed. For the 3 \# measuring point, its relative difference is about $3 \%$ when rotational speeds are $200 \mathrm{r} / \mathrm{min}$ and $250 \mathrm{r} / \mathrm{min}$, and its relative difference is about $8 \%$ at other speeds. The main reason for the above phenomenon is that the theoretical model cannot completely simulate the actual test situation. During the test, the loading screw exerts a force on pad, and there is a contact friction between loading module and pad, which will restrict pad's movement, thus affecting eccentricity and attitude angle of the pad. These factors are not considered in the theoretical model bearing. However, the impact is not significant, so it is only reflected in some working conditions.
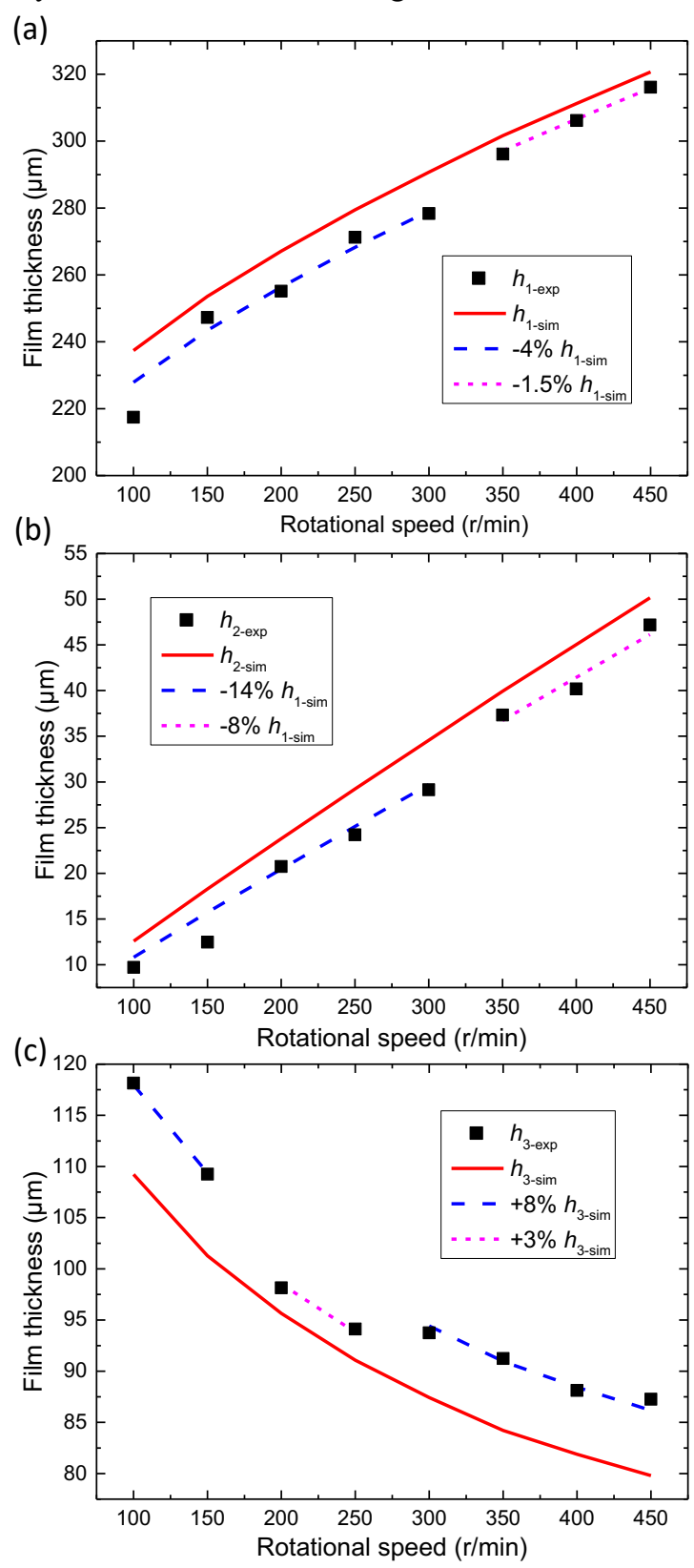
FIG. 13. Comparison of experimental and simulated values of film thickness: (a) 1\# measuring point; (b) $2 \#$ measuring point; (c) $3 \#$ measuring point

\section{Prediction of key parameters of bearing film thickness distribution}

Bearing deformation and wear are not considered. When the working condition is unchanged, film thickness values and position of the three measuring points are brought into formula (1), then three linear equations can be established, and radius clearance $c b$, eccentricity $\varepsilon$ and attitude angle $\theta_{0}$ can be obtained by solving the equations.

The load is $4 \mathrm{kN}$, attitude angle and eccentricity at different speeds are predicted. At the same time, mean predicted value of bearing radius clearance at different speeds is $0.5619 \mathrm{~mm}$, and its measured value is $0.56 \mathrm{~mm}$, which are close. According to the predicted eccentricity and radius clearance, the minimum film thickness can be calculated. The predicted value and simulated value at the rotational speed of $350 \mathrm{r} / \mathrm{min} \sim 550 \mathrm{r} / \mathrm{min}$ are shown in Figure 14. The minimum and maximum values of relative difference between predicted and simulated values of attitude angle are about $3.1 \%$ to $8.3 \%$. The minimum and maximum values of relative difference between predicted and simulated values of eccentricity are about $0.1 \%$ to 0.2 $\%$. Except for the rotational speed at $350 \mathrm{r} / \mathrm{min}$, relative difference between predicted and simulated values of the minimum film thickness at other speeds is about $2.8 \%$ $7.1 \%$. Relative difference at the rotational speed of 350 $\mathrm{r} / \mathrm{min}$ is $13.3 \%$, but the absolute difference is only about 1 $\mu \mathrm{m}$. Prediction accuracy of eccentricity has a great influence on prediction result of film thickness.

In general, the prediction method has high feasibility and accuracy. According to the predicted value of bearing film thickness distribution, bearing lubrication state can be monitored in real time, which will provide a feasible method for monitoring and warning of bearing lubrication failure. Future works will focus mainly on modifying the lubrication model according to the experiment results, such as identifying journal bending bearing deformation.

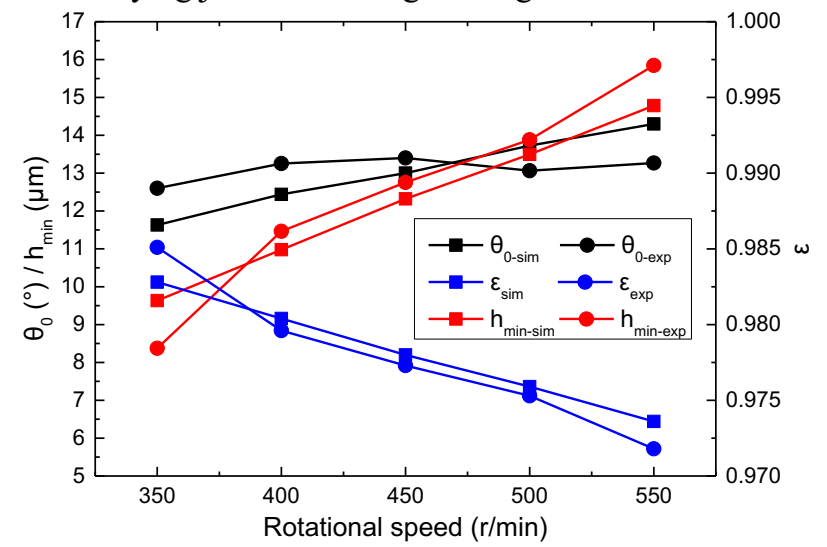

FIG. 14. Comparison of predicted and simulated values of key parameters of film thickness distribution
Method for measuring film thickness distribution of bearing based on the ultrasonic is proposed. The measuring device is calibrated and a measurement experiment of bearing's film thickness distribution is carried out. Some significant conclusions have been obtained.

(1) The full circumferential measurement method can obtain full circumferential film thickness distributions in real time, but it requires high precision and stability of wireless telemetry. The prediction method based on limited measuring points can predict key parameters of film thickness distribution, and it's simple to implement.

(2) For calibration results, when film thickness ranges from $10 \mu \mathrm{m}$ to $100 \mu \mathrm{m}$, the relative error of most recognition values is less than $\pm 5 \%$. When the range is $100 \sim 150 \mu \mathrm{m}$, the relative error is less than $\pm 3 \%$. The relative error is less than $5 \%$ in the range of $2.5 \sim 7.5 \mu \mathrm{m}$. When it is greater than $7.8 \mu \mathrm{m}$, the recognition result is very sensitive to the error of reflection coefficient, and the relative error is large.

(3) Experimental value of bearing film thickness is close to simulated value based on the hydrodynamic lubrication theory. Eccentricity, attitude angle, and the minimum film thickness of bearing are predicted based on film thickness values of three measuring points. Their relative difference is less than $8.3 \%$ in most working conditions, which verifies the measurement method has high accuracy.

\section{DATA AVAILABILITY STATEMENT}

The data that support the findings of this study are available from the corresponding author upon reasonable request.

\section{ACKNOWLEDGMENTS}

This project is supported by the National Key Research and Development Project (No. 2018YFE0197600) and the European Union's Horizon 2020 Research and Innovation Programme RISE under grant agreement no. 823759 (REMESH).

\footnotetext{
${ }^{1}$ M. Torbacke, Å. K. Rudolphi, E. Kassfeldt, John Wiley \& Sons (2014).

${ }^{2}$ W. Ouyang, X. Zhang, Y. Jin, et al., Shock and Vib., 1 (2018).

${ }^{3}$ W. Litwin., Tribol. Int., 103, 352 (2016).

${ }^{4}$ F.M. Kuang, X.C. Zhou, J. Huang, et al., Wear, 426, 760 (2019).

${ }^{5}$ Q.W. Huang, X.P. Yan, C. Zhang, et al., Ocean Eng., 178, 48 (2019).

${ }^{6}$ Y. Henry, J. Bouyer, M. Fillon, Tribol. Int., 120, 299 (2017).

${ }^{7}$ J. Bongaerts, J. Day, C. Marriott, et al., J Appl. Phys., 104, 014913 (2008).

${ }^{8}$ S.C. Richards, A.D. Roberts, J Appl. Phys., 25, 76 (1992).

${ }^{9}$ A. Ponjavic, J. Wong, RSC Advances, 4, 20821 (2014).

${ }^{10}$ X.X. Liang, X.P. Yan, W. Ouyang, et al. Surf. Topogr.- Metrolo., 7, 045010 (2019).

${ }^{11}$ F. Zhang, W. Ouyang, H. Hong, et al., Tribol. Int., 88, 228 (2015).

${ }^{12}$ J. Jiao, W. Liu, J. Zhang, et al., Mech. Syst. Signal Pr., 35, 69 (2013).

${ }^{13}$ T. Pialucha, C.C.H. Guyott, P. Cawley, Ultrasonic, 27, 270 (1989).

${ }^{14}$ K. Zhang, T. Wu, Q. Meng, et al., Int. J Adv. Manuf., 94, 3209(2018).

${ }^{15}$ R.S. Dwyer-Joyce, B.W. Drinkwater, C.J. Donohoe, P. Roy. Soc. AMath. Phy., 459, 957 (2003).

${ }^{16}$ R.S. Dwyer-Joyce, P. Harper, B.W. Drinkwater, Tribol. Lett., 17, 337 (2004).
} 
${ }^{17}$ R.S. Dwyer-Joyce, T. Reddyhoff, J. Zhu, J TRIBOL.-T. ASME, 133, 1 (2011).

${ }^{18}$ P. Dou, T. Wu, Z. Luo, J TRIBOL.-T. ASME, 141, 031702 (2019).

${ }^{19}$ R.S. Dwyer-Joyce, P. Harper, J. Pritchard, et al., P. I. Mech. Eeg. A-J Pow., 220, 619 (2006).

${ }^{20}$ J.Y. Gong, Y. Jin, Z.L. Liu, et al., Tribol. Int., 129, 390 (2019). 